\title{
Status and perspectives of the ASDEX Upgrade gas inlet system
}

\author{
Thomas Härtl $^{\mathrm{a}}$, Volker Rohde ${ }^{\mathrm{a}}$, Vitus Mertens ${ }^{\mathrm{a}}$ and ASDEX Upgrade team \\ ${ }^{a}$ Max Planck Institute for Plasma Physics, Boltzmannstr. 2, 85748 Garching, Germany
}

\begin{abstract}
Installed in the late 1980s the gas inlet system of ASDEX Upgrade was state of the art at the time of commissioning. However, after more than 20 years of operation a redesign was needed to take the growing complexity of experimental scenarios into account and to replace out-dated components.

A conceptual design based on a study of operational requirements as well as available resources was developed and the gas inlet system was renewed step by step during planned maintenance shutdowns of AUG over the last years. The improvement comprises the building of a gas supply station, construction of a system for the gas assignment, relocation of the operational gas inlet and inside-vessel gas guiding as well as a redesign of the communication with the control system. Since the campaign 2014 the renewed system is in routine operation.
\end{abstract}

\section{Keywords: ASDEX Upgrade, gas inlet system, tokamak fuelling, piezo electric valve, variable gas supply}

\section{Introduction}

Present-day fusion devices using metal plasma facing components require a versatile gas inlet system for operation. Supplying the plasma discharge with different gases, e.g. hydrogen isotopes for fuelling, nitrogen or noble gases for radiation cooling and miscellaneous impurity gases for plasma control are tasks of such systems.

Next to these plasma operational duties, wall conditioning (glow discharge cleaning, GDC) and wallcoating (boronisation) are requirements of the gas inlet system (GES), but are not described in this paper.

The GES of ASDEX Upgrade (AUG) was installed during the early stages of the device in the late 1980s. This design was based upon the operational experience from the GES of AUG's predecessor. The system was state of the art at the time of commissioning. However, changing requirements and a growing complexity in the experimental scenarios meant the GES became insufficiently fit for purpose. This was apparent in an unsuitable distribution of gas inlet positions and limitations in supplying them with the necessary gas species needed for challenging plasma investigations and avoiding mutual interference with diagnostics. Use of system modifications enabled the operation of AUG but was aligned with high maintenance costs and increased resource requirements as well as the necessity for planning days in advance.

Furthermore, after more than 20 years of operation functional assurance and spare part supply of single components gets increasingly difficult to maintain.

Analysing requirements from recent years showed that up to ten separate gases or gas mixtures are used within experimental campaigns. Our experiences handling the operational gases within the building and below ground level, with the associated safety controls, led to a preference for future outdoor storage. Increases in reliability and an associated reduction in the requirement for maintenance gave rise to an advantageous lowering of the number of gas cylinders needed. New piezoelectric valves (PV) come with a high price and lengthy procurement lead-time. For this reason, new PVs, additional to the 20 existing piezoelectric valves used for the fast gas inlet into the vacuum vessel, were not procured.

A conceptual design relevant to the described requirements was developed using $\mathrm{PVs}$ located in a suitably well distributed manner. Additionally the construction allows all gas introduction locations to be supplied with all relevant species and mixtures with a fast changeover time as well as low costs of operation. Being absolutely necessary for the operation of AUG, GES rebuilding work was carried out during planned maintenance shutdowns of AUG.

\section{Description and overview GES}

The parts of the new GES can be grouped according technical duty: gas supply, gas assignment, operational gas inlet, gas guiding and control. The complete system is controlled by a Simatic S7 PLC.

\subsection{Gas supply}

The supply station is installed outside of the building that accommodates the experimental hall, laboratories and offices. The supply station is a roofed open-air construction. This reduces the mandatory required equipment for explosion prevention and simplifies safety issues like supervision and delivery of replacement gas bottles. It consists of ten gas control panels each comprising of a pressure regulator, overpressure relief valve and automated shut-off valve. Each unit allows the connection of all types of gases, with the exception of toxic or corrosive gases. Apart from during experiments, the shut-off valves are kept closed. This maintains the inventory of gases within the building to a minimum. Via tubes of about 25 metre length (inner pipe diameter $8 \mathrm{~mm}$ ) the supply unit is connected to the valve-matrix. 


\subsection{Assignment of gas to PVs - Valve-Matrix}

Allocation of available gases to a specific PV is accomplished by a matrix consisting of 12 x 20 pneumatic cut-off valves (Fig.1). This design enables the operator to independently assign and change the gas species for one or more PV without any mutual interference with others. The matrix was built from 20 identical linear valve assemblies consisting of 12 single valves welded together. These are connected to the 10 supply-lines with VCR-fittings. This reduces the risk of leakage from flange connections but still allows the ability to provide maintenance of worn and defective parts by replacement of a whole linear valve assembly. To stabilize the typical operational PV pressure of 4 bar(abs), an electronic pressure regulator adjustable by PLC is installed in each supply-line, a gas type independent capacitance absolute pressure transducer exists in each valve line.

Two pumping lines are installed next to the gascarrying tubes. This enables the evacuation of individual sections of the matrix for the purpose of gas changes or leak testing. Equipped with a combination of dry forepump (multi-stage Roots pump) and turbo molecular pump (TMP) an attainable pressure of $<10^{-2} \mathrm{~Pa}$ is measurable in the gas lines. This ensures a residual gas concentration after a change of species comparable to typical suppliers delivered gas purities.

To compromise between a high conductance for evacuation and a low duct volume to economise on gas resources, an inner pipe diameter of approximately $8 \mathrm{~mm}$ (Tube: $10 \times 1.0 \mathrm{~mm}$ or $1 / 8$ ”), was chosen resulting in a pipe volume of about 2 litres. For quality and safety reasons only welding or metal sealing was used for connecting the up to $40 \mathrm{~m}$ long stainless steel pipe installations. The gas lines for the PVs are fuelled all the time, minimising the supply pressure drop of the PVs during a plasma discharge to a few $\mathrm{hPa}$.

With this matrix it is possible to execute a change of gas species for one or several PVs within less than 15 minutes and therefore in between two consecutive plasma discharges.

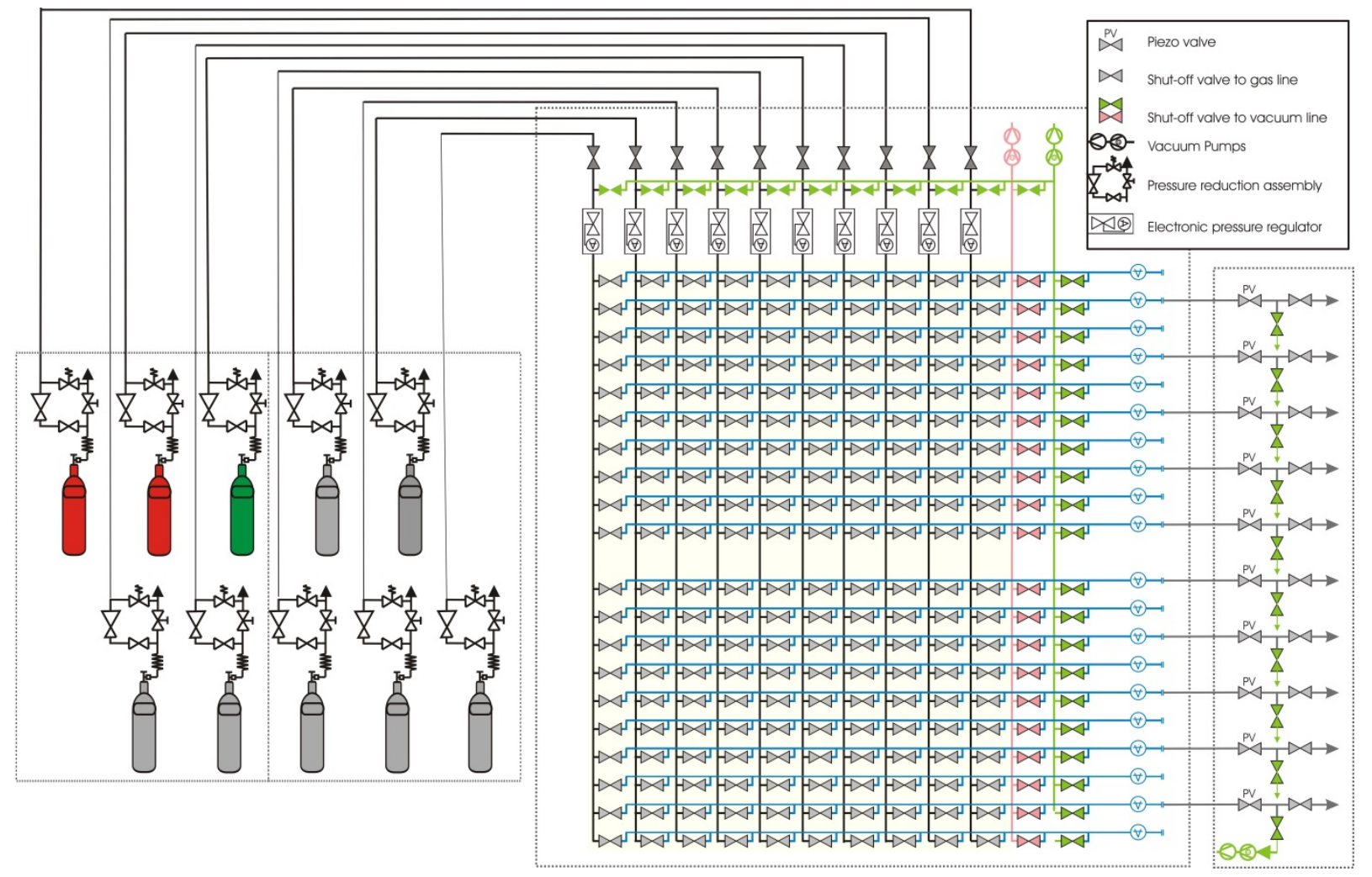

Fig. 1. Overview of the AUG fast gas inlet system. Outdoor pressure regulator units for 10 different gases (left). ValveMatrix to assign gas species to PV supply lines (middle). Typical valve group at the torus (schematic figure only) (right).

\subsection{Fast gas inlet valves}

The fast gas valves (PV) feeding the plasma discharge (Fig. 2) are specially designed by General Atomics to operate at fusion devices and are capable of operating within strong magnetic fields [1]. The actuating element - the piezo ceramics - equipped with a Viton seal is controlled by a voltage of up to $250 \mathrm{~V}$, correspondingly opens valve seating. The gas passes through a $1.5 \mathrm{~mm}$ diameter capillary tube with a small downstream pressure sensor. Every PV is directly connected to a dedicated valve controller unit, which is matched and prepared for the operational characteristics of the attached valve, to overcome the sticking effect of the Viton seal to ensure a reliable fast opening and regulate the driving voltage to reach and follow the target values of the flow as fast as possible. Based on the signal of the pressure sensor the closed loop circuit of the controller guaranties a long term highly reproducible gas flow. This is calibrated with the usually needed gas 
species. Thereby as many defined gas pulses at a specific flow rate are allowed to ingress into the non-pumped and actively temperature controlled AUG vessel until stable pressure of some $\mathrm{Pa}$ (measured with a high accuracy capacitive diaphragm gauge) is achieved. This procedure is repeated for up to 15 different flow rates. Thereby an accuracy of about $5 \%$ is reachable over the entire flow range. The maximum gas flow rate depending on gas species reaches up to $50 \mathrm{~Pa} \mathrm{~m}^{3} \mathrm{~s}^{-1}\left(3,6 \mathrm{e} 19\right.$ atom s$\left.{ }^{-1}\right)$ for Hydrogen and $8 \mathrm{~Pa} \mathrm{~m}^{3} \mathrm{~s}^{-1}$ for Argon. The closed loop of the valve-system reaches a rise and fall time of $\sim 1 \mathrm{~ms}$ in total [1].

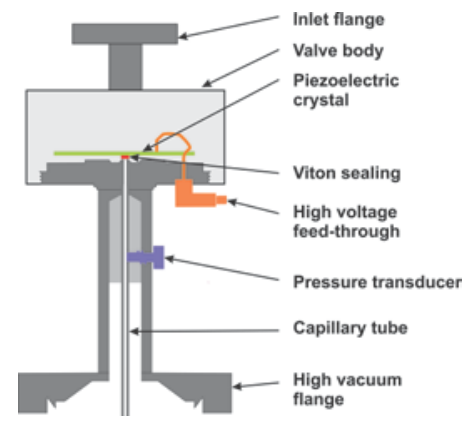

Fig. 2. Shows a schematic sectional chart of a PV.

\subsection{Cabling of PV and Controller}

Due to the relocation of some of the PVs on the Torus new cabling between the PV and the controller was necessary. For ease of installation and to avoid magnetic loops caused by the standard use of two cables, a completely new custom-built cable was designed with assistance of a notable manufacturer and subsequently procured (Fig. 3). It includes the wires for powering the pressure sensor (gy, pi), the pressure signal (bn, gn, with additionally shielding) and two lines for accessory current supply (wh, ye) as well as the wire to activate the piezo crystal of the valve (RG178). To enhance the firesafety it is completely manufactured from halogen-free materials.

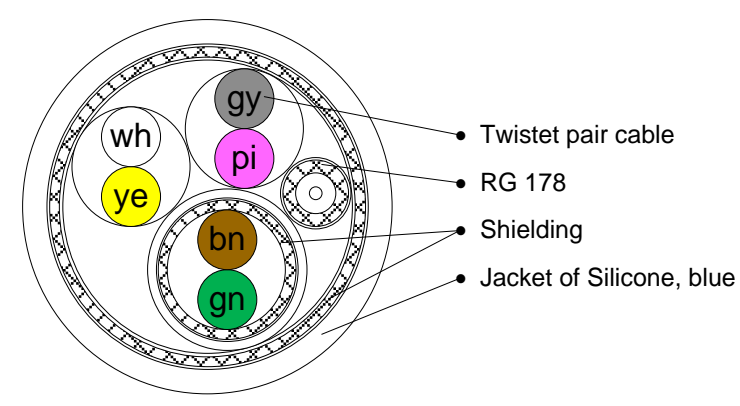

Fig. 3. Cross section of the custom-built cable

The qualification testing at PV02 (Co02A) has proven functionality of the new cable concept and exceeded expectations with respect of signal quality improvement. Fig. 4 shows the flow signal of the PV with the old and new cable connection during comparable discharges. Based on this very positive experience, the cables of all valves were replaced, using the opportunity to choose cable conduits not required to accommodate high-voltage or high frequency cable.

As the controllers are situated outside the experimental hall for better accessibility an average cable length of up to $70 \mathrm{~m}$ is necessary for each valve for a continues installation from plug to plug without additional connector blocks.

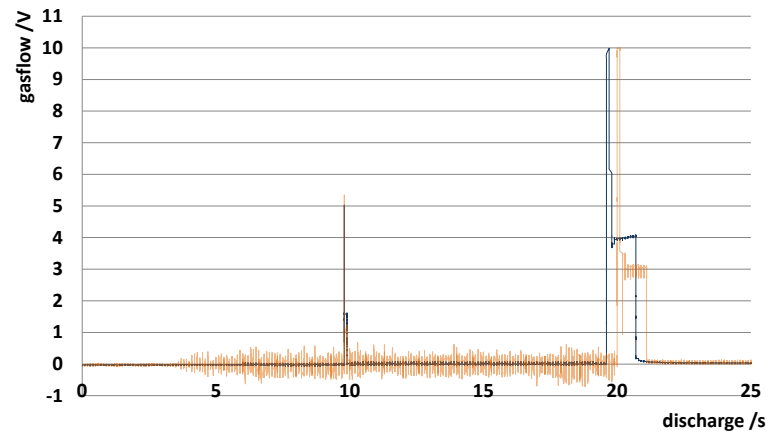

Fig. 4. Comparison of the signal quality of the PV Co02A with old cabling (orange) and the new one (blue) during two similar plasma discharges.

\subsection{Gas feed in-vessel tubing}

The gas feed in the lower divertor region suffered from the high pumping speed of the divertor cryo-pump (CP), which pumped most of the gas before reaching the plasma. To overcome these difficulties, conduits were installed connecting from the PVs, bypassing the CP and allowing the gas to ingress directly into the private flux region of the plasma. Installation started with the so called DuX-Valves (Du01X, Du05X, Du09X, Du13X). Each valve conduit is equipped with two outlets for a homogenous distribution. Due to the encouraging results with this solution also the new installed DuB-valves (Du01B, Du05B, Du09B, Du13B) in the lower divertor and the FoA-valves (Fo02A, Fo07A, Fo10A, Fo14A) in the upper divertor were equipped with a conduit, however using just one outlet each.

In Table 1 an overview of all installed PVs is shown, giving the technical PV number, mounting position, gas supply line, physical designation and additional information.

The technical implementation was made using a combination of Teflon hose inside the AUG ducts and stainless steel tubes in the plasma facing region. An inner diameter of $6 \mathrm{~mm}$ was chosen as a compromise reaching appropriate conductance and fast time response of $30 \mathrm{~ms}$ to $100 \mathrm{~ms}$. The overall length of the conduit is about 3 meters for the DuX-valves and 4 meters for the DuB and FoA valves. The shut-off valves to the torus as well as the evacuation valves were replaced by new designed valve groups with minimised dead volumes. These divertor located valves currently have the least mutual interference with diagnostics and are presently the standard gas feed for AUG operation. 
Table 1. Overview of installed PVs

\begin{tabular}{|r|r|r|r|r|r|}
\hline PV \# & Port & Sector & Gas supply & UVD Name & Comment \\
\hline PV01 & & & VM_01 & & n/a \\
\hline PV02 & Co & 02 & VM_02 & CFCo02A & \\
\hline PV03 & Fo & 02 & VM_03 & CFFo02A & in-vessel tube \\
\hline PV04 & A & 13 & VM_04 & CFA13B & in-vessel tube \\
\hline PV05 & A & 03 & VM_05 & CFA03B & \\
\hline PV06 & Fo & 07 & VM_06 & CFFo07A & in-vessel tube \\
\hline PV07 & Du & 05 & VM_07 & CFDu05X & in-vessel tube \\
\hline PV08 & Du & 05 & VM_08 & CFDu05B & in-vessel tube \\
\hline PV09 & Co & 10 & VM_09 & CFCo10A & ICRF antenna \\
\hline PV10 & Du & 01 & VM_10 & CFDu01B & in-vessel tube \\
\hline PV11 & Fu & 09 & VM_11 & CFDu09B & in-vessel tube \\
\hline PV12 & Fo & 10 & VM_12 & CFFo10A & in-vessel tube \\
\hline PV13 & Fo & 14 & VM_13 & CFFo14A & in-vessel tube \\
\hline PV14 & A & 03 & VM_14 & CFA03C & special Gl \\
\hline PV15 & A & 13 & VM_15 & CFA13A & \\
\hline PV16 & A & 03 & VM_16 & CFA03A & \\
\hline PV17 & Du & 09 & VM_17 & CFDu09X & in-vessel tube \\
\hline PV18 & Du & 13 & VM_18 & CFDu13X & in-vessel tube \\
\hline PV19 & Du & 13 & VM_19 & CFDu13B & in-vessel tube \\
\hline PV20 & Du & 01 & VM_20 & CFDu01X & in-vessel tube \\
\hline
\end{tabular}

For feeding gas in the midplane three valves are installed at the port A03. For the use of rare or expensive gases one of them is designed to be used with a local gas cylinder thus minimising gas losses during a change of species. Two valves are mounted at the port A13; one of those is equipped with a tube for fluctuation imaging measurements. For investigations of the influence of gas puffing on ICRF coupling a multi-point outlet is installed directly between limiter and faraday-cup of the antenna structure [2]. The valve located at the port Co02 is mainly used for pre-fill.

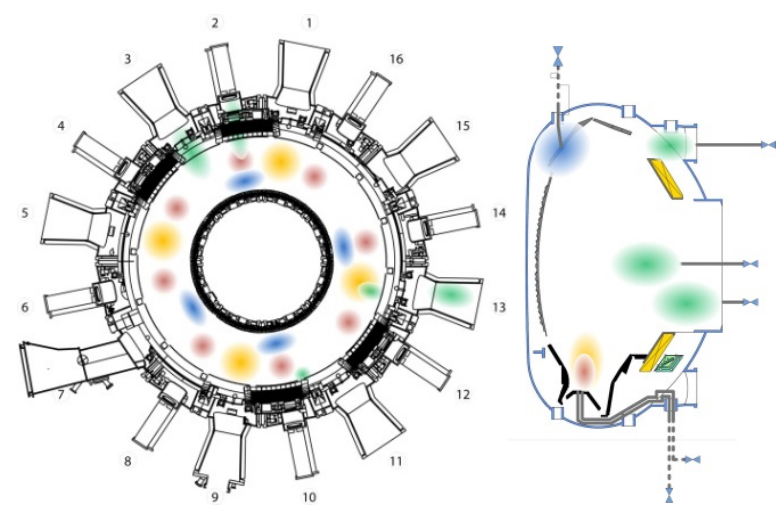

Fig. 5. Visualisation of the gas inlet positions inside the vacuum vessel. View from top (left) and vertical cross section with schematic projection of all position (right). Colouring corresponds to Table 1.

\section{Operational control of PVs}

AUG plasma operation is managed by the Discharge Control System (DCS) which also enables feedback controlled requisition of gas flows [3]. Communication between GES and DCS has been enhanced substantially; this now enables direct access to all PVs by the experiment leaders. Therefore operational status of PVs and corresponding valve groups as well as connected gas species is available in a newly introduced front end.

\section{Operational experience}

All modifications were operable for the 2014 experimental campaign and were used for multifaceted experiments. Thereby occasionally more than ten PVs were used within a single plasma discharge operated in feed forward as well as feed-back controlled mode of operation.

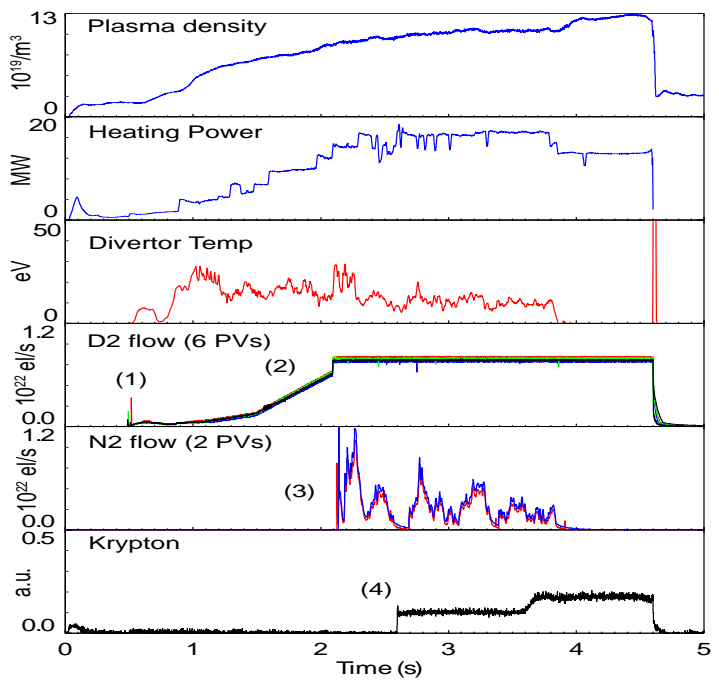

Fig. 6. Graph of shot\#30450, high density, high radiation scenario discharge. Started by prefill (1), $\mathrm{D}_{2}$ density was built-up with six PVs (2). Simultaneously, Divertor temperature was controlled by $\mathrm{N}_{2}$ blowing with 2 PVs (3). Plasma shaping was controlled by an additional Krypton inlet (4).

\section{Outlook}

The positions of tube guided gas inlets inside the vessel seem to be well suited for present experimental operation. However, the versatile use with different gas species requires a continuing calibration of the PVs.

Presently the procedure for a changeover in the gas supplying valve matrix is carried out manually. For minimising risk of maloperation leading to impurity of the gases, automation is planned over the medium term.

\section{Acknowledgement}

This work has been carried out within the framework of the EUROfusion Consortium and has received funding from the European Union's Horizon 2020 research and innovation programme under grant agreement number 633053. The views and opinions expressed herein do not necessarily reflect those of the European Commission.

\section{References}

[1] S. C. Bates, K. H. Burrell, Fast gas injection system for plasma physics experiments, Review of Scientific 
Instruments 55 (1984) 934.

[2] V. Bobkov, Influence of gas injection location and magnetic perturbations on ICRF antenna performance in ASDEX Upgrade, AIP Conf. Proc. 1580, 271 (2014).

[3] W. Treutterer et al., ASDEX Upgrade Discharge Control System - A real-time plasma control framework, Fusion Engineering and Design 89 (2014) 146-154. 